\title{
Smart Sensor Network System based on ZigBee Technology to Monitor Grain Depot
}

\author{
Santoshkumar \\ Assistant Professor, \\ Electrical Engineering \\ Department, \\ SDM College of Engineering \\ and Technology, \\ Dharwad, India.
}

\author{
Vishal Hiremath \\ Electrical Engineering \\ Department, \\ SDM College of Engineering \\ and Technology, \\ Dharwad, India.
}

\author{
Rakhee K \\ Electrical Engineering \\ Department, \\ KLE College of Engineering \\ and Technology, \\ Belgaum, India.
}

\begin{abstract}
The nation's economy and quality of life depends on the agricultural yield and the storage of food grains has been an age long practice with cultivators and traders. Good storage facilities are important to the farmers all over the world as they ensure household and community food security until the next harvest. Though considerable losses occur in the field, both before and during harvest, the greatest losses are noticed during storage. Considerable losses both in quality and quantity of food-grains take place in storage due to a number of factors.

A Smart Sensor System is proposed to monitor grains in storage depots. The damage of grains can be controlled by monitoring the parameters like temperature, humidity and light which influence the storage of grains. The insects and fungus are the two prominent factors which damage and spoil the grains in the depot and are controlled by temperature, humidity and light. The low cost, low power smart wireless sensor network system is designed to monitor the parameters influencing the storage of grains in depot. The open source technology-ARDUINO (open electronics prototype and open source) is used to develop the system.
\end{abstract}

\section{General Terms}

Wireless Sensor Networks, Sensors

\section{Keywords}

Arduino, temperature, humidity, light, ZigBee

\section{INTRODUCTION}

The temperature, humidity and light are key parameters in monitoring the grains stored in grain depot. The variation in the temperature and humidity may lead to grain damage and might spoil the grains. The insects and fungus are the two factors which damage and spoil the grains in the depot and are controlled by temperature, humidity and light.

The optimum temperature for the insects is $25^{\circ} \mathrm{C}$ to $32^{\circ} \mathrm{C}$. The reduction in the development of the insects occurs when the temperature is less than $14^{\circ} \mathrm{C}$ and greater than $42^{\circ} \mathrm{C}$

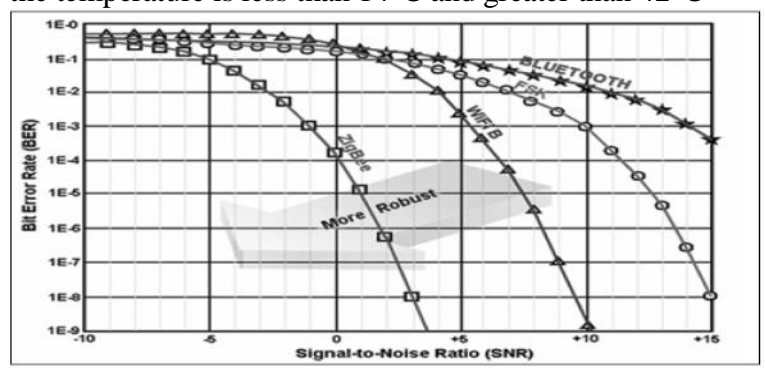

Figure 1. ZigBee v/s other Wireless Technologies respectively. For the insects to perish, the temperature levels should be maintained below 5 and above 45 degrees centigrade respectively. The optimum relative humidity for most species of insects is $70 \%$ with minimum of 25 to $40 \%$ and maximum of 80 to $100 \%$. Very few species survive in extremely dry conditions. The light can reduce the movement and fertility of flying insects like moths. This results in less destruction of grains. Also, there is a loss of vitamins through the action of sunlight and temperature [6].

The grain damage by fungi can be reduced when the grain is stored at moisture content below $14 \%$ and the temperature is less than $20^{\circ} \mathrm{C}$ and above $40^{\circ} \mathrm{C}$ respectively.

This problem can be solved by employing Sensor Network that would enable users to monitor the required factors such as temperature, humidity and light. Sensor Networks offer many attractive low cost solutions to monitor these conditions. The sensor node is designed and developed for the sensor network applications, as the commercial nodes come with fixed design and are costly.

The main aim of Sensor Networks is to sense any data from the desired location, transmit the same. The sensed parameters can be viewed at the receiver side in a personal computer. Sensor network is a combination of various sensors that are positioned to monitor. A sensor provides extra functions beyond those necessary for generating a correct representation of the sensed quality such as signal conditioning, signal processing and decision-making and alarm functions. The smart sensor network system is designed using open source technology-ARDUINO.

\section{RELATED WORK}

After reviewing many articles, there are very few papers that discuss about monitoring the grain depot. In the available papers only two parameters like temperature and humidity are considered [1]. There is no mention of light, which is a prominent parameter and is responsible for reduction in the movement and fertility of flying insects like moths. Also the grain damage due to fungi is not clear.

\section{WIRELESS SENSOR NETWORKS}

Wireless sensor network (WSN) is used for data acquisition, processing and data distribution. A WSN system consists of nodes and a base station. A node is composed of a processor, local memory, sensors, radio and battery and a base station is responsible for receiving and processing data collected by the nodes. They perform collective activities due to limited resources such as battery, processor and memory [2] [3] [4] [5]. Grain depot monitoring is interesting application where the conditions such as temperature, humidity and light are to 
be monitored. In such applications, the sensors are fixed and WSN should guarantee real time monitoring. The accuracy of monitoring and controlling the minute details of a distantly located station is a technological challenge. Earlier the wireless networks used RF and Bluetooth technologies and later replaced by ZigBee.

The Figure. 1 shows how ZIGBEE takes over other wireless technologies. As the Signal-Noise-Ratio (SNR) increases, the bit error rate decreases. That is, for higher signal-to-noise ratios, the bit error rate is very less. Though this trend is observed in all the wireless technologies, only ZIGBEE shows a prompt decrease of bit-error rate even for less signal-tonoise ratios. This implies that, even in the presence of high noise strength almost equalling the signal strength, the system can trace the required signal eliminating the bit-errors. This gives a very robust performance.

A WSN consists of generally two main parts: Data Acquisition Network and Data Distribution Network. The Figure 2.shows a simplified block diagram WSN System.

The main aim of the Wireless Sensor Networks is to sense any data from the desired location, transmit the same through wireless network. The block diagram of wireless sensor network consists of two main parts, transmitting side and receiving side respectively. At the transmitting side, various sensors and controllers are connected with the FREEDUINO and XBEE. At the receiving side the transceiver and the microcontroller are present along with the display device. The sensed parameters with their exact precision values are transmitted to the observing station through Wireless Communication and details are monitored by the administrator. When any of the parameter measured is found to be above a threshold value either an Alarm is generated to avoid the damage of grains

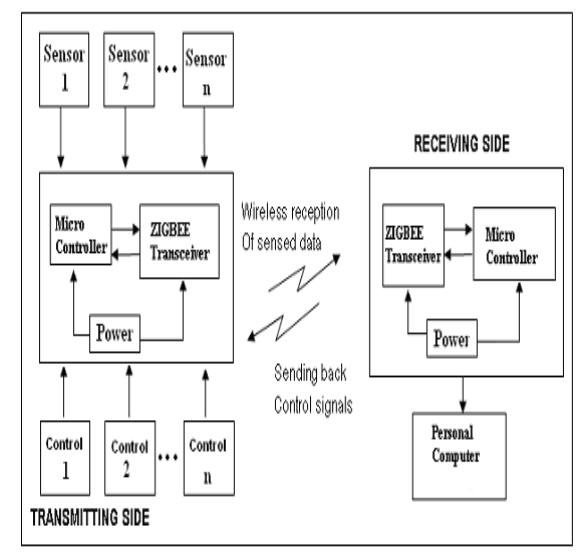

Figure 2.WSN block diagram

\section{ZIGBEE PROTOCOL OVERVIEW}

ZIGBEE is a specification for wireless personal area networks (WPANs) operating at $868 \mathrm{MHz}, 902-928 \mathrm{MHz}$, and $2.4 \mathrm{GHz}$. A WPAN is a personal area network (a network for interconnecting an individual's devices) in which the device connections are wireless. Using ZIGBEE, devices in a WPAN can communicate at speeds of up to $250 \mathrm{Kbps}$ while physically separated by distances of up to 50 meters in typical circumstances and greater distances in an ideal environment. ZIGBEE is based on the 802.15 specification approved by the Institute of Electrical and Electronics Engineers Standards Association (IEEE-SA).

\subsection{IEEE 802.15.4}

The ZigBee protocol uses IEEE 802.15.4 specifications as its Medium Access Layer (MAC) and Physical Layer (PHY). The IEEE 802.15.4 defines a total of three frequency bands of operations: $2.4 \mathrm{GHz}, 915 \mathrm{MHz}$ and $868 \mathrm{MHz}$. Each frequency band offers a fixed number of channels. The bit rate of the protocol depends on the selection of frequency of operation. The $2.4 \mathrm{GHz}$ band provides $250 \mathrm{kbps}, 915 \mathrm{MHz}$ provides 40 kbps and $868 \mathrm{MHz}$ provides a $20 \mathrm{kbps}$ data rate. The actual data throughput would be less than the specified bit rate due to the packet overhead and processing delays. The maximum length of an IEEE 802.15.4 MAC packet is 127 bytes. Each packet consists of header bytes and a 16-bit CRC value. The 16-bit CRC value verifies the frame integrity. In addition, IEEE 802.15.4 optionally uses an acknowledged data transfer mechanism. This makes sure that a frame is in fact delivered.

\subsection{Network Configurations}

A ZigBee wireless network may assume many types of configurations. A star network configuration consists of one coordinator node (a "master") and one or more end devices ("slaves"). The coordinator is a special variant of a Full Function Device (FFD) that implements a larger set of ZigBee services. The end devices may be FFD or a Reduced Function Device (RFD). An RFD is the smallest and simplest ZigBee node. It implements only a minimal set of ZigBee services. In a star network, all end devices communicate to the coordinator only. The coordinator periodically begins with a superframe identified as a beacon frame and all nodes in the network are expected to synchronize to this frame. Each node is assigned a specific slot in the superframe during which it is allowed to transmit and receive its data. The Figure 3.shows Network configuration. ZigBee networks can be ad-hoc, meaning that a new network is formed and unformed as needed. In a star network configuration, end devices would always search for a network before they can perform any data transfer. A new network is first established by a coordinator. On start-up, a coordinator searches for other coordinators nearby and if none is found, it establishes its own network and selects a unique 16-bit PAN ID. Once a new network is established, one or more end devices are allowed to associate with the network. A typical ZigBee node may support multiple features and functionality. In order to reduce cost, a ZigBee node uses only one radio channel and multiple endpoint/interfaces to create multiple virtual links or channels. One ZigBee node supports 31 endpoints (numbered $0-31$ ) and 8 interfaces (numbered 0-7). The endpoint 0 is reserved for device configuration and endpoint 31 is reserved for broadcasts only. This leaves a total of 30 endpoints for application use. A typical ZigBee node would also have many attributes. A collection of attributes is called a cluster. Each cluster is assigned a unique cluster ID in the entire network. Each cluster may have up to 65,536 attributes. The ZigBee protocol also defines a term called profile. A profile is synonymous to the description of a distributed application.

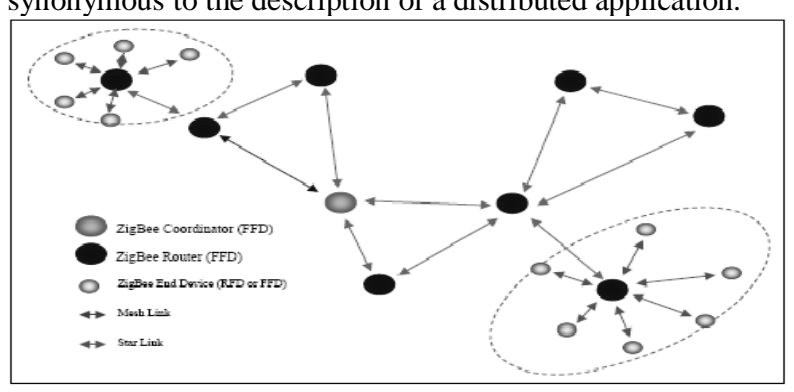

Figure 3.Network Configuration 


\section{METHODOLOGY}

A very systematic approach was considered for the overall design of the project, in which three parameters were monitored. The temperature, humidity and light sensors were used to monitor the grain depot. The node is designed for the increased battery life and the ZigBee technology supports same and is shown in Figure 4 below.

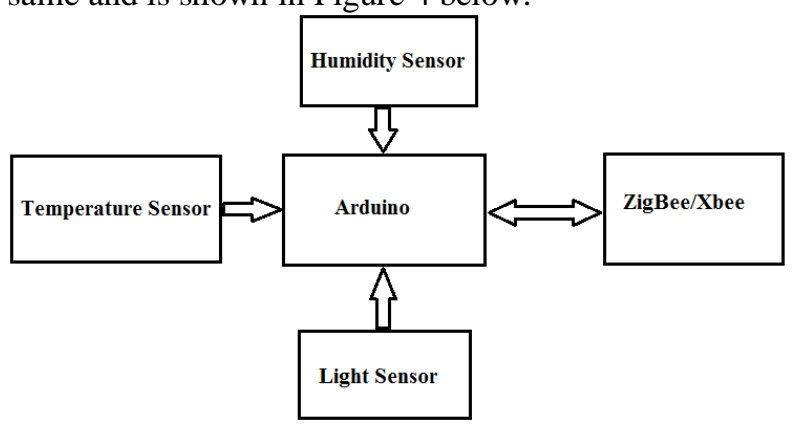

Figure 4. Sensor Node

\subsection{Grain Depot}

Good storage facilities are very important as they provide food security until next harvest. The losses occurring in the grain storage depots can be controlled by monitoring the temperature, humidity and light in the depot.

\subsection{Hardware Description}

\subsubsection{Microcontroller}

The Freeduino board uses the ATMega328, was selected as the microcontroller for this project. This was an ideal selection, as the processor is extremely strong and cost efficient. An input voltage ranging from $0-5 \mathrm{~V}$ is required, which corresponds with the humidity sensor. An on-board 10bit analog to digital converter (ADC), aids in the digitization of the analog signal acquired from the sensor. The Arduino has a serial port to allow communication with the computer. The USB connection from the computer goes directly onto the Arduino board, where a USB to serial converter then allows communication to occur. PIC's also contained 10- bit ADCs, however they had operating voltages ranging from $1.8-5.5 \mathrm{~V}$.

\subsubsection{Temperature Sensor}

The temperature within a store is affected by the sun, the cooling effect of radiation from the store, outside air temperature, heat generated by the respiration of both the food in store and any insect pest present. The lower and upper temperature threshold values were set to $14^{\circ} \mathrm{C}$ and $42^{\circ} \mathrm{C}$ respectively to control the damage done by insects and fungi.

The LM35 temperature sensor produces an analog voltage directly proportional to temperature with an output of 1 mill volt per $0.1^{\circ} \mathrm{C}(10 \mathrm{mV}$ per degree $)$. The sketch (program) converts the analogRead values into milli volts and divides this by 10 to get degrees. The temperature sensor is shown in Figure 5

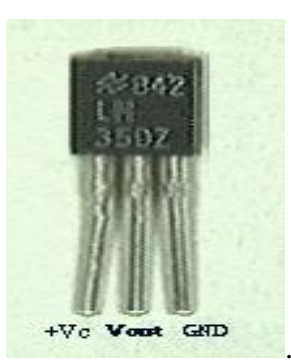

Figure 5.Temperature Sensor

\subsubsection{Humidity Sensor}

All insects require moisture to survive and multiply. If the moisture content in a product that is to be stored is low, insects cannot grow. Therefore the moisture should be minimum and the threshold value set is $9 \%$ which results in reduced normal activity and development. The humidity sensor is designed using op-amp and is shown in Figure 6. below.

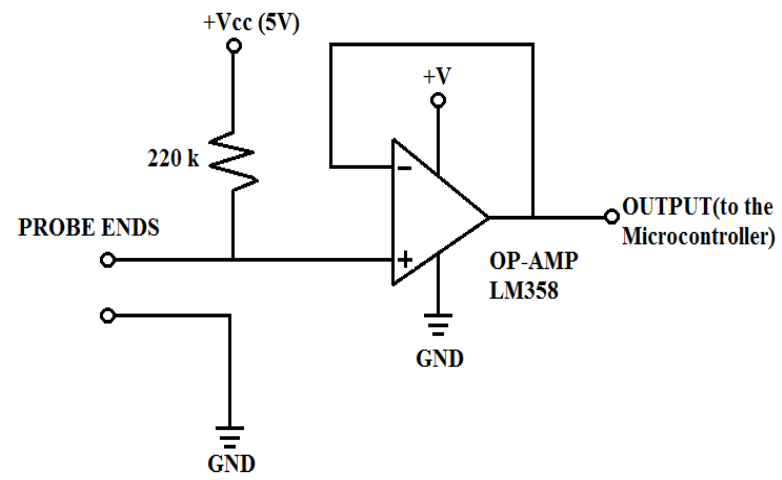

\subsubsection{Light Sensor}

Figure 6.Humidity sensor

Stored product insects reaction to light is more varied. Few insects are attracted towards light while most of the insects avoid it preferring a subdued light or darkness. The lower and upper light threshold values were set to 50 and 90 Lux respectively. Light sensor was designed using op-amp and is shown in Figure 7. below.

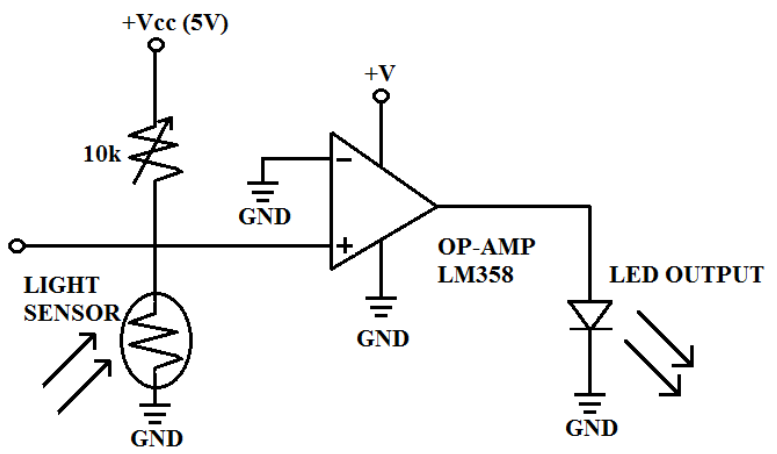

Figure 7.Light Sensor

\section{DESIGN ISSUES}

The nodes were deployed in the depot and the parameters were sensed and the mesh networking was considered with five nodes. The alarm signal is sent by the corresponding node which senses the values below or above the thresholds. The battery life is increased as nodes enter into sleep mode if the values are well within threshold limits. The minimum temperature threshold value is set to $14^{\circ} \mathrm{C}$ and the minimum humidity threshold value is set to $9 \%$.

The software design consists of two main sections: Transmitting side and Receiving side. At the transmitting side, various sensors are connected to the FREEDUINO. Continuously the values are monitored and the details of distantly located sensors are stored in the base station as data base. At the Base Station (receiving side) the transceiver and the FREEDUINO are present along with the display device. The sensed values are sent to the observing station. The flow charts of the developed algorithm for the WSN system for grain depot and the base station are shown below in figures Figure 8. and Figure 9. 


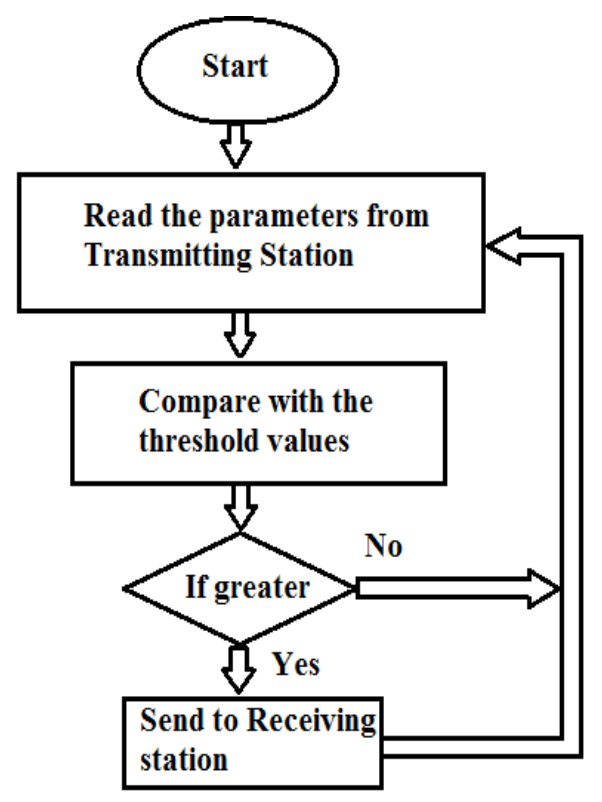

Figure 8. Flow chart for Transmitting Station

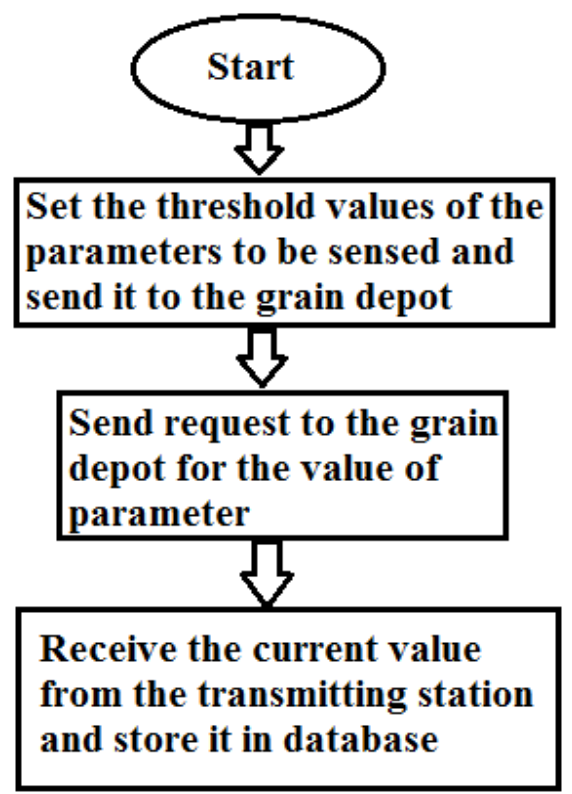

Figure 9. Flow chart for Receiving Station

\section{RESULTS}

The various laboratory experiments were performed to validate the proposed system and the smart wireless sensor system was fixed in the grain depot to monitor the parameters influencing the grain damage. The system developed showed promising results. The measured temperature, humidity and light are shown in figures Figure 10, 11 and 12 respectively.

\section{CONCLUSION}

The developed prototype wireless sensor network system using open source technology-ARDUINO has proven to successfully acquire accurate measurements for the above mentioned parameters like, temperature, humidity and light. The alarm is sent to the observing station if the value received at the observing station is above the threshold value. So, this helps us to monitor the grain depot and help us to prevent the damage done to the stored grains in depots and thus helping in the growth of the nation and improving the quality of life.

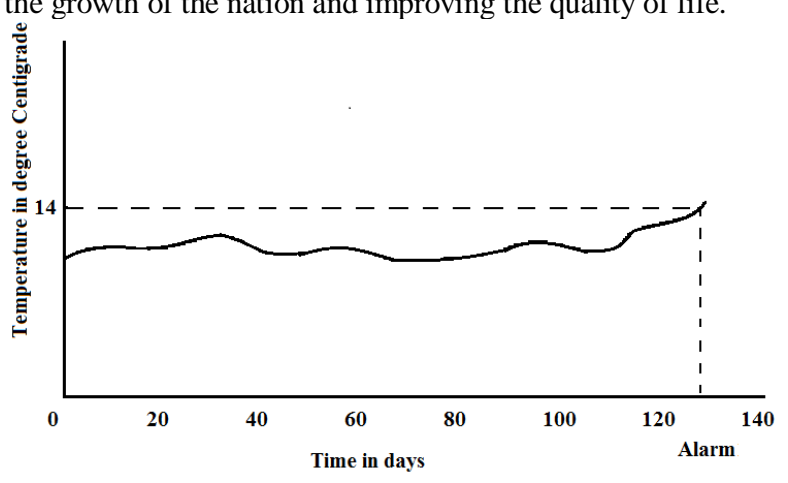

Figure 10. Temperature

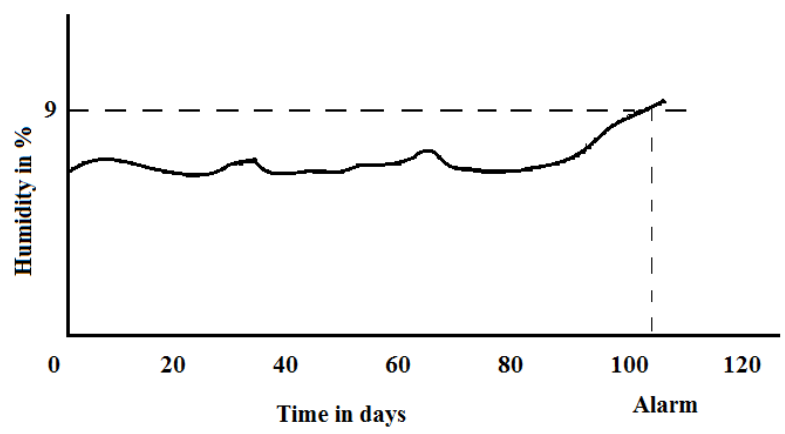

Figure 11. Humidity

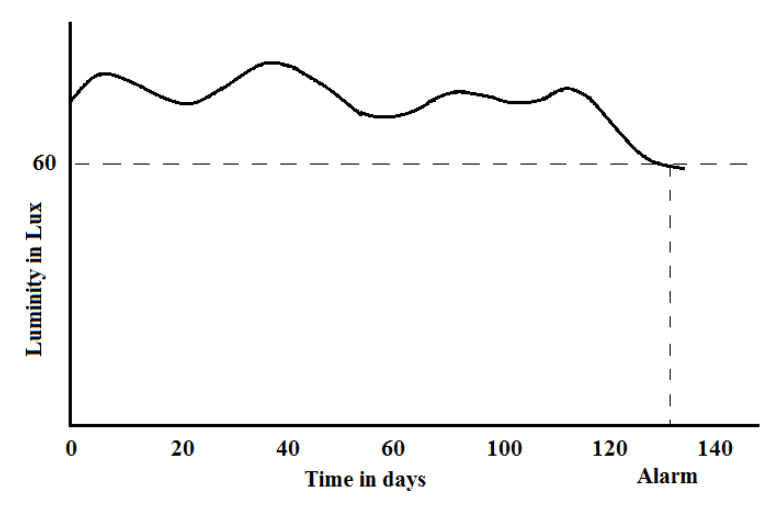

Figure 12. Light

\section{REFERENCES}

[1] Jianguang Jia, Jingming Kuang, Zunwen He and Yuhang $\mathrm{Mu}$, "Design of Monitor System for Grain Depots Based on Wireless Sensor Network", IEEE International Conference on Mechatronics and Automation held at China during August 9-12, 2009.

[2] Santoshkumar, Vishal Hiremath, “ Design and Development of Wireless Sensor Network System to Monitor Parameters Influencing Freshwater Fishes" International Journal of Computer Science and Engineering (IJCSE), Vol.4, No.6, 2012.

[3] Santoshkumar, Dr.D.G.Wakde, "Development of Wireless Sensor Node for Precision Agriculture", IEEE International Conference on Computational Intelligence and Computing Research (IEEE-ICCIC-2011) at Kanyakumari, India. 
[4] Santoshkumar, Kelvin C. Chelli, Suresh Chavhan, "Development of Wireless Sensor Node to Monitor Poultry Farm", accepted as full paper in Second International Conference on Advances in Information Technology and Mobile Communication-AIM2012 held at Bangalore, India during April 27-28,2012 and proceedings published in Springer-Verlag, LNCS.

[5] Santoshkumar and Rakhee K "Design and development of Wireless Sensor Network System based on ZIGBEE for Greenhouse" National Conference on Advances in Wireless Communications (NCACW-2010) Shegaon, India.
[6] C. Chong and S.P. Kumar, "Sensor Networks: Evolution, Opportunities, and Challenges," Proc. IEEE, vol. 91, no. 8,2003.

[7] D. Culler, D. Estrin, and M. Srivastava, "Overview of Sensor Networks," Computer, vol. 37, no. 8, 2004.

[8] F. Akyildiz et al., "A Survey on Sensor Networks," IEEE Communications Magazine, vol. 40, no. 8, 2002. 